contain carbon tetrachloride, the brass squirts so universally carried are of less use than mascots. This substance will not extinguish petrol fires. At best, it may localize the combustion. It yields stifling clouds of black soot and twice its own vapour. volume of chlorine. It is itself toxic.

As a young man, I worked as an assistant both to R. V. Wheeler and to H. B. Dixon, and it has occurred to me to follow up the latter's investigation of the former's discovery of the combustion-inhibiting influence of the iodides. One of many reasons for the choice of methyl iodide for the initial experiments was its physical resemblance to carbon tetrachloride. Chemically and physiologically it is very different. It is safer and pleasanter in use; and it does not yield noxious or corrosive end-products.

It was found that in all cases where carbon tetra. chloride was effective, methyl iodide was equally effective with one fifth of the quantity. Both were better sprayed than squirted, and the pumps and syringes needed non-greasy lubrication. The crucial experiment was then tried of spraying it on to a water-borne layer of petrol on fire. Up to forty square feet, garden syringes were used. Over greater areas a pump was desirable. This test gave a complete failure to carbon tetrachloride, a saturated water solution of ammonium iodide and to methylene di-iodide. These substances burnt in the flames. Under a spray of methyl iodide, however, the petrol flames broke, wilted and withered. There was no smothering or swamping; the effect resembled that of a policeman's hand on traffic. Moreover, the petrol can at will be repeatedly rekindled and requenched until it is made safe or exhausted.

Tray-fires of ether, methylated spirits, and white spirit, which could not be extinguished by carbon tetrachloride, were swiftly quelled by methyl iodide.

Some hundreds of comparisons made, less picturesquely, with a flash-point apparatus contain no single exception to this overwhelming superiority of methyl iodide.

This fact has immense possibilities in social consequences-the price, of course, must fall just as the price of D.D.T. fell. What it amounts to in physicel chemistry seems to be a fascinating example of how combustion-chains may be affected by iodine atoms freshly liberated from a single valency. The iodine liberated in the burning of methylene di-iodide would clearly seem to have a different mode of existence.

16 Heaton Road,

Withington,

Manchester 20.

Nov. 8.

\section{A Simple Moving-Boundary Apparatus}

IN the conventional types of apparatus for the measurement of transference numbers in electrolyte solutions by the moving-boundary method, elaborate devices are used to achieve the initial formation of a reasonably sharp boundary ${ }^{1,2}$. It seems surprisingly to have escaped notice so far that a sharp 'sheared' boundary can be formed satisfactorily and with less trouble by the use of a single three-way tap of good quality.

The apparatus used by us, illustrated in the accompanying figure, has been designed for the measurement of the transference number of hydrogen ion in hydrochloric acid solutions by a descending boundary in the tube $A$, using lithium chloride as the

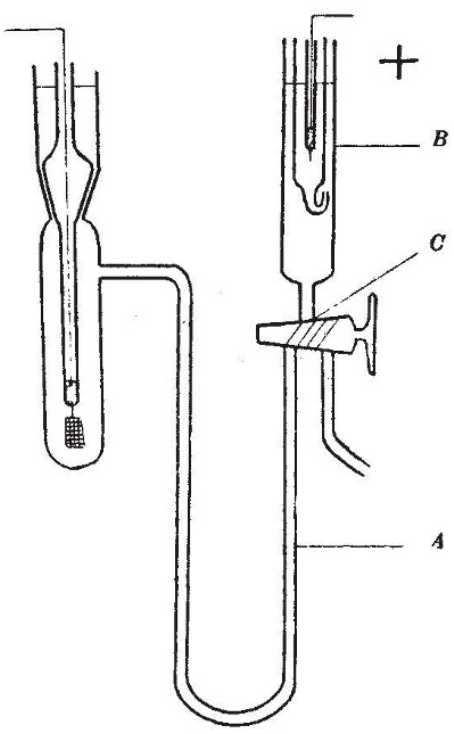

'indicator' electrolyte. The adaptation of the principle to other measurements is obvious.

The whole apparatus is first filled with the hydro. chloric acid solution with the tap in the position connecting $A$ and $B$, after which the anode vessel is emptied (by turning the tap through $180^{\circ}$ ). $B$ can then be rinsed through with the indicator solution at leisure. After turning the tap through $90^{\circ}, B$ is filled with the indicator solution and, on connecting $A$ and $B$ again by turning the tap to the initial position, a sharp boundary is formed at $C$ the velocity of which may be measured after it has passed through the tap.

We wish to thank Messrs. E. C. A. Horner and A. D. Jenkins for experimental assistance.

$$
\text { E. G. BAXTER }
$$$$
\text { V. GOLD }
$$

Department of Chemistry,

King's College,
London, W.C.2.

Nov. 27.

1 Macinnes and Longsworth, Chem. Rev., 11, 179 (1932) (review of then existing types).

3 Hartley and Donaldson, Trans. Farad. Soc., 33, 458 (1937).

\section{Elminius modestus in the Netherlands}

RECENTLY, Jones ${ }^{1}$ remarked that the barnacle Elminius modestus Darwin, if introduced into France and Holland, is likely to become particularly troublesome for the oyster culture industry. It may be worth while to state that the species seems to be already fairly common in the southern part of the North Sea coast of the Netherlands.

As Bishop ${ }^{2}$ stated that the barnacle was taken from the hull of the M.V. Empire Wansbeck, which had been plying between Harwich and the Hook of Holland, the species might be expected here. To direct attention to its probable occurrence, a note giving particulars of the barnacle was inserted in a stencilled periodical devoted to the study of the seashore, and the Leyden Museum soon received material of Elminius from Scheveningen and its surroundings and from Serooskerke in Zeeland. The first specimens on flotsam were collected on September 19, 1946 ; the first specimens on piles were obtained on October 